\title{
A note on circulatory systems: Old and new results Circulatory systems
}

\section{Kliem, Wolfhard; Pommer, Christian}

\section{Published in:}

Zeitschrift fuer Angewandte Mathematik und Mechanik

Link to article, DOI:

10.1002/zamm.201600056

Publication date:

2017

Document Version

Publisher's PDF, also known as Version of record

Link back to DTU Orbit

Citation (APA):

Kliem, W., \& Pommer, C. (2017). A note on circulatory systems: Old and new results: Circulatory systems. Zeitschrift fuer Angewandte Mathematik und Mechanik, 97(1), 92-97. https://doi.org/10.1002/zamm.201600056

\section{General rights}

Copyright and moral rights for the publications made accessible in the public portal are retained by the authors and/or other copyright owners and it is a condition of accessing publications that users recognise and abide by the legal requirements associated with these rights.

- Users may download and print one copy of any publication from the public portal for the purpose of private study or research.

- You may not further distribute the material or use it for any profit-making activity or commercial gain

- You may freely distribute the URL identifying the publication in the public portal

If you believe that this document breaches copyright please contact us providing details, and we will remove access to the work immediately and investigate your claim 


\title{
A note on circulatory systems: Old and new results
}

\author{
Wolfhard Kliem* and Christian Pommer \\ DTU Compute, Department of Applied Mathematics and Computer Science, Building 324, Technical University of \\ Denmark, DK-2800 Kgs. Lyngby, Denmark
}

Received 18 March 2016, revised 14 April 2016, accepted 21 June 2016

Published online 2 September 2016

Key words Ciculatory systems, stability, indefinte damping, gyroscopic damping.

MSC (2010) 34D20, 34D10

It is astonishing that after more than half a century intensive research in the area of non-conservative systems of second order differential equations still new interesting results appear, see [4]. In that paper an old stability criterion by Metelitsyn [8] and Frik [9] was reinvented. We shortly repeat this result in order to emphasize that the criterion is sufficient but not necessary for stability. Afterwards we concentrate on circulatory systems with purely imaginary eigenvalues and investigate the influence of indefinite damping. Finally the possibility of stabilizing circulatory systems by gyroscopic forces will be commented. Examples will demonstrate the developed theory.

\section{Introduction}

This paper deals mainly with second order systems of linear differential equations of the form

$$
M \ddot{x}+(K+N) x=0, \quad M=M^{T}>0, K=K^{T}>0, N=-N^{T} .
$$

Such MKN-systems are sometimes called pseudo-conservative [1], since they show some similarity to conservative systems with $N=0$. We prefer the name circulatory systems [2,3]. Recently a paper appeared about the influence of damping terms $D \dot{x}$ with $D \geq 0$ on circulatory systems, and on more general non-conservative systems as well, [4]. In our paper we will firstly recapitulate the most important of these results including some additional comments and secondly we will show the influence of indefinite damping on circulatory systems (1) with only purely imaginary eigenvalues. A few words concerning modeling in Mechanics with a circulatory matrix $N$ may be adequate. Stiffness matrices with a non-symmetrical part $N$ are well-known in rotor dynamics due to internal damping or to non-symmetrical steam flow in turbines, due to follower forces in elasto-mechanics, in pipe lines etc. They can lead to self-excited vibrations and therefore to instability of the system. A recent example is the investigation of disc brakes [5]. Moreover, circulatory forces can stabilize an unstable conservative system and destabilize a stable conservative system, see e.g. [2,3]. Indefinite damping in mechanical systems should be addressed shortly as well. E. g. sliding bearings [6] and cutting of of metals [7] can be modelled with indefinite damping which can lead to self-excited vibrations. If the eigenvalues of a system are symmetrical with respect to both axes we say that the system possesses Hamiltonian symmetry. Circulatory systems have Hamiltonian symmetry. We will show how an unstable circulatory system under certain circumstances can be stabilized by adding a gyroscopic term $G \dot{x}$ with $G=-G^{T}$. To this end we use a theory developed in [15]. With help of this theory we can transform a $I D G K N$-system with Hamiltonian symmetry into an isospectral circulatory $I K_{0} N_{0}$-system to facilitate the investigation of stability.

\section{Some known results}

In [4] stability of more general linear non-conservative MDGKN-systems of the form

$$
M \ddot{x}+(D+G) \dot{x}+(K+N) x=0, M=M^{T}, D=D^{T}, K=K^{T}, G=-G^{T}, N=-N^{T}, M, K>0
$$

was under consideration as well. To this end a theorem by Metelitsyn [8] and Frik [9], also mentioned in [3], was reinvented. Without loss of generality we assume $\mathrm{M}=\mathrm{I}$. Then stability of system (2) can be completely understood with help of the eigenvalue problem

$$
\left(\lambda^{2} I+\lambda(D+G)+K+N\right) u=0, \quad u \neq 0 .
$$

\footnotetext{
* Corresponding author E-mail: wolfhard@kliem.dk
} 
The eigenvalues $\lambda$ are the roots of the characteristic polynomial $\operatorname{det}\left[\lambda^{2} I+\lambda(D+G)+K+N\right]$, and if all eigenvalues have negative real parts, system (2) is asymptotically stable. Marginal stability is characterized by semi-simple eigenvalues on the imaginary axis (at least one) together with all the other eigenvalues having negative real parts. Pre-multiplying (3) by the conjugate transposed $u^{*}$ of the eigenvector $\mathrm{u}$ and normalizing by $u^{*} u=1$, we receive (one equation for each eigenvector)

$$
\lambda^{2}+(d+i g) \lambda+(k+i n)=0
$$

where the coefficients $d=u^{*} D u, i g=u^{*} G u, k=u^{*} K u$, in $=u^{*} N u$ are the Rayleigh quotients of the matrices $D, G$, $K$, and $N$, respectively. Their numerical values are limited by smallest and largest eigenvalues of the respective matrices. (For example $\lambda(D)_{\text {min }} \leq d \leq \lambda(D)_{\max },-|\lambda(G)|_{\max } \leq g \leq|\lambda(G)|_{\text {max }}$, etc.). If we now claim that both roots of (4) have negative real parts we receive the following theorem:

Metelitsyn-Frik

System (2) is asymptotically stable if

$$
d>0 \wedge d^{2} k+d g n-n^{2}>0 .
$$

By taking advantage of the properties of the Rayleigh quotients these inequalities lead to

$$
d_{\min }>0 \wedge d_{\min }\left(d_{\min } k_{\min }-g_{\max } n_{\max }\right)-n_{\max }^{2}>0 .
$$

Although these two conditions (5) were already derived in [4], we repeated shortly the procedure in order to point out that the criterion is only sufficient for asymptotic stability, but not nescessary, since the eigenvalue $\lambda$ is one of the roots of Eq. (4), but the other root need not to be an eigenvalue. Actually, it is more an exception than a rule that both roots of (4) are eigenvalues. Discussion and examples can be found in [10]. Any I $D G K N$-system with $K>0$ and $D>0$ will be asymptotically stable if dissipative and/or potential forces are sufficiently large. It is a crucial point that the inequalities of the criterion are not necessary for asymptotic stability. To claim sufficient large $d_{\text {min }}$ may lead to an undesirable large damping. Therefore Theorem 2 in [4] is of importance, where asymptotic stabilization of a $I K N$-system with purely imaginary eigenvalues can be achieved by a damping $D>0$ with arbitrarily small norm.

\section{Indefinite damping in circulatory systems with imaginary eigenvalues}

In order to investigate the influence of indefinite damping matrices on circulatory systems with purely imaginary eigenvalues we recall the concept of symmetrizability. A real square matrix $C$ is called (real) symmetrizable if any of the four equivalent conditions is satisfied:

1. There exists a non-singular matrix $W$ such that $W^{-1} C W$ is symmetric.

2. $C$ possesses only real eigenvalues and a full set of eigenvectors.

3. $C$ is the product of two symmetric matrices, one of which is is positive definite. (If both symmetric matrices are positive definite, then $W^{-1} C W>0$ and all eigenvalues of $C$ are positive.)

4. $C$ becomes symmetric when multiplied by a suitable positive definite matrix.

The circulatory systems $I K N(1)$ under consideration are connected to the eigenvalue problem $\left(\lambda^{2} I+K+N\right) u=0$, where we assume $\lambda \in i \mathbb{R}$. This is a necessary (but not sufficient) condition for the $I K N$-system to be stable. Moreover, if we add damping, necessary conditions for asymptotic stability of the $I D K N$-system are trace $(D)>0$, trace $\left(K^{-1} D\right)>0$, and $\operatorname{det}(K+N)>0$, [3]. The following investigation is divided into 2 cases:

i) The matrix $C=K+N$ is real symmetrizable and both symmetric matrices in condition 3. are positive definite. Then also $W^{-1} C W>0$ and the $I K N$-system is completely marginally stable.

ii) The matrix $C=K+N$ is not symmetrizable. Then the $I K N$-system is not stable.

\section{Case i)}

In [11] the influence of an indefinite damping matrix on a conservative and stable IK-system was investigated by a perturbation approach. The perturbation analysis assumes small damping terms compared to the conservative forces described by $K$. We will show that a similar procedure works for a circulatory $I K N$-system.

Let the damping matrix $D=D^{T}$ depend smoothly on the vector $p=\left(p_{1}, p_{2}, \ldots, p_{n}\right)$ of real parameters (for example $\left.D=\operatorname{diag}\left(p_{1}, p_{2}, \ldots, p_{n}\right)\right)$. Consider $p$ as a point in the parameter space. At $p=p_{0}$ we have $D\left(p_{0}\right)=0$ and system $I K N$ posesses a purely imaginary eigenvalue $\lambda_{0}=i \omega_{0}$ with a full set of real eigenvectors. We want to study the behavior of the eigenvalue in the vicinity of the initial point $p_{0}$. To this end we consider a parameter variation $p=p_{0}+\epsilon e$, where 
$e=\left(e_{1}, . ., e_{n}\right)$ is a direction vector of unit norm, $|e|=1$, and $\epsilon>0$ is a small perturbation parameter. Hence we obtain

$$
D=D_{0}+\epsilon D_{1}+\epsilon^{2} D_{2}+\ldots
$$

where

$$
D_{0}=D\left(p_{0}\right), \quad D_{1}=\sum_{i=1}^{n} \frac{\partial D}{\partial p_{i}} e_{i}, \quad D_{2}=\frac{1}{2} \sum_{i, j=1}^{n} \frac{\partial^{2} D}{\partial p_{i} \partial p_{j}} e_{i} e_{j}, \quad \ldots
$$

Then the eigenvalue, the right eigenvector $u$ and the left eigenvector $v$ of the IDKN-system can be expanded in power series of $\epsilon$

$$
\lambda=\lambda_{0}+\epsilon \lambda_{1}+\epsilon^{2} \lambda_{2}+\ldots, \quad u=u_{0}+\epsilon u_{1}+\epsilon^{2} u_{2}+\ldots, \quad v=v_{0}+\epsilon v_{1}+\epsilon^{2} v_{2}+\ldots
$$

We introduce the matrix valued functions

$$
L(\lambda)=\lambda^{2} I+\lambda D+K+N, \quad L_{1}(\lambda)=\sum_{i=1}^{n} \frac{\partial L}{\partial p_{i}} e_{i}=\lambda_{0} D_{1} .
$$

Inserting (7) and (8) into $L(\lambda) u=0$ and comparing expressions with the same power of $\epsilon$ leads to

$$
\lambda_{1}=-\frac{v_{0}^{T} L_{1} u_{0}}{2 \lambda_{0} v_{0}^{T} u_{0}}=-\frac{v_{0}^{T} D_{1} u_{0}}{2 v_{0}^{T} u_{0}} .
$$

$\lambda_{1}$ is real since the nominator and denominator both are real. Therefore the eigenvalue $\lambda$ has a negative real part in the vicinity of $\lambda_{0}$, if $\lambda_{1}<0$ which means stability.

Example 3.1. Consider the circulatory system $I K N$ with

$$
K=\left[\begin{array}{ll}
4 & 2 \\
2 & 3
\end{array}\right], \quad N=\left[\begin{array}{rr}
0 & 1 \\
-1 & 0
\end{array}\right] .
$$

Then matrix $C=K+N$ is real symmetrizable with positive eigenvalues and eigenvectors and system $I K N$ is completely marginally stable. We choose $\lambda_{0}=2.3028 i$ with right eigenvector

$$
u_{0}=\left[\begin{array}{l}
0.9172 \\
0.3983
\end{array}\right]
$$

and the left eigenvector

$$
v_{0}=\left[\begin{array}{l}
0.6089 \\
0.7933
\end{array}\right]
$$

and a direction vector $e=\left(e_{1}, e_{2}\right)=\frac{1}{\sqrt{5}}(2,-1)$. Then

$$
D(p)=\left[\begin{array}{cc}
p_{1} & 0 \\
0 & p_{2}
\end{array}\right], D_{1}=\frac{\partial D}{\partial p_{1}} e_{1}+\frac{\partial D}{\partial p_{2}} e_{2}=\frac{1}{\sqrt{5}}\left[\begin{array}{rr}
2 & 0 \\
0 & -1
\end{array}\right]
$$

and

$$
\lambda_{1}=-\frac{v_{0}^{T} D_{1} u_{0}}{2 v_{0}^{T} u_{0}}=-0.2048
$$

implies stability. If

$$
D=\left[\begin{array}{rr}
0.02 & 0 \\
0 & -0.01
\end{array}\right],
$$

we get $\epsilon=\frac{\sqrt{5}}{2} \cdot 0.02=0.02236$ and $\lambda=\lambda_{0}+\epsilon \lambda_{1}+o(\epsilon)=2.3028 i-0.00458+o(\epsilon)$.

Respectively, for $\lambda_{0}=1.3028 i$ we receive

$$
u_{0}=\left[\begin{array}{r}
-0.7933 \\
0.6089
\end{array}\right], \quad v_{0}=\left[\begin{array}{r}
-0.3983 \\
0.9172
\end{array}\right]
$$

and

$$
\lambda_{1}=-\frac{v_{0}^{T} D_{1} u_{0}}{2 v_{0}^{T} u_{0}}=-0.0188
$$

implies stability. Finally $\lambda=\lambda_{0}+\epsilon \lambda_{1}+o(\epsilon)=1.3028 i-0.00042+o(\epsilon)$. 
Example 1 showed stability of a I $D K N$-system for an indefinite damping matrix $D$. But a positive definite damping can also cause instability if $K+N$ is symmetrizable as the next example from [4] shows.

Example 3.2. Consider the $I K N$-system with

$$
K=\left[\begin{array}{ll}
3 & 0 \\
0 & 2
\end{array}\right], \quad N=\left[\begin{array}{cc}
0 & 0.499 \\
-0.499 & 0
\end{array}\right]
$$

Assume

$$
D_{1}=\frac{1}{\sqrt{13}}\left[\begin{array}{ll}
3 & 0 \\
0 & 2
\end{array}\right] .
$$

Then we receive for eigenvalue $\lambda_{0}=1.5991 i$ and respective right and left eigenvectors according to (10) $\lambda_{1}=-1.4436$ which means stability. But for eigenvalue $\lambda_{0}=1.5711 i$ we receive $\lambda_{1}=0.7502$ which implies instability, as also shown graphically in [4].

Actually in [4] theorem 1 it was shown that for any circulatory IKN-system with only purely imaginary eigenvalues there exists a damping matrix $D \geq 0$ such the the resulting $I D K N$-system is unstable.

\section{Case ii)}

We assume that at $p=p_{0}$ the $I K N$-system posesses a double eigenvalue with only one right eigenvector $u_{0}$ and one left eigenvector $v_{0}$. Then there excists a Jordan chain of lenght 2, [12]

$$
L_{0} u_{0}=0, \quad L_{0} w_{0}=-\frac{\partial L}{\partial \lambda} u_{0}, \quad L_{0}=\lambda_{0}^{2} I+K+N, \quad v_{0}^{T} L_{0}=0, \quad \frac{\partial L}{\partial \lambda}=2 \lambda_{0} I
$$

where $w_{0}$ is a generalized right eigenvector. The $I K N$-system is not stable and a solution includes a secular term. I order to study the behavior of eigenvalues in the vicinity of $p=p_{0}$ we assume like in case i) a variation $p=p_{0}+\epsilon e$. According to the perturbation theory developed in $[13,14]$ for case ii), expansions of eigenvalues and eigenvectors contain fractional powers of $\epsilon$

$$
\begin{aligned}
& \lambda=\lambda_{0}+\epsilon^{1 / 2} \lambda_{1}+\epsilon \lambda_{2}+\ldots, \quad u=u_{0}+\epsilon^{1 / 2} u_{1}+\epsilon u_{2}+\ldots, \\
& v=v_{0}+\epsilon^{1 / 2} v_{1}+\epsilon v_{2}+\ldots
\end{aligned}
$$

With the same definitions for $L, L_{1}$, and $D_{1}$ as in case i) we receive

$$
\lambda_{1}^{2}=-\frac{\lambda_{0} v_{0}^{T} D_{1} u_{0}}{v_{0}^{T} \frac{\partial L}{\partial \lambda} w_{0}+v_{0}^{T} u_{0}} .
$$

Since $L_{0}$ is singular, a generalized eigenvector $w_{0}$ as a solution of $L_{0} w_{0}=-2 \lambda_{0} u_{0}$ only exists if $v_{0}^{T} u_{0}=0$. Then $w_{0}$ is purely imaginary and therefore also $\lambda_{1}^{2}$ is purely imaginary and

$$
\lambda=\lambda_{0}+\epsilon^{1 / 2} \lambda_{1}+o\left(\epsilon^{1 / 2}\right)=\lambda_{0} \pm \sqrt{\epsilon \lambda_{1}^{2}}+o\left(\epsilon^{1 / 2}\right) .
$$

This shows that the double eigenvalue $\lambda_{0}=i \omega_{0}$ bifurcates into two eigenvalues, one of which has a positive real part, which means instability by flutter for all kind of damping matrices $D(p)$. We should mention that if in case ii) the matrix $K+N$ depends on a load parameter $q$, a small variation $\delta q$ of this parameter causes flutter instability as well.

Example 3.3. Consider the $I K N$-system

$$
K=\left[\begin{array}{ll}
4 & 2 \\
2 & 3
\end{array}\right], \quad N=\frac{\sqrt{17}}{2}\left[\begin{array}{rr}
0 & 1 \\
-1 & 0
\end{array}\right] .
$$

The system $I K N$ has a double eigenvalue $\lambda_{0}=1.87083 i$ with only one right eigenvector $u_{0}$ and one left eigenvector $v_{0}$ and a generalized eigenvector $w_{0}$ given by

$$
u_{0}=\left[\begin{array}{r}
0.99251 \\
-0.12218
\end{array}\right], \quad v_{0}=\left[\begin{array}{l}
0.12218 \\
0.99251
\end{array}\right], \quad w_{0}=\left[\begin{array}{c}
4659.74822 i \\
-574.55556 i
\end{array}\right]
$$

If we choose the direction $e=\frac{1}{\sqrt{5}} \cdot(2,-1)$ in the parameter space and the indefinite damping matrix

$$
D=\left[\begin{array}{rr}
0.02 & 0.00 \\
0.00 & -0.01
\end{array}\right]
$$


then this leads to $\epsilon=\frac{\sqrt{5}}{2} \cdot 0.02=0.02236$ and $\lambda_{1}^{2}=0.08964 i$. Finally we receive

$$
\lambda \simeq \lambda_{0}+\epsilon^{1 / 2} \lambda_{1}=\left\{\begin{array}{r}
0.03166+1.90249 i \\
-0.03166+1.83917 i
\end{array},\right.
$$

which shows a bifurcation of the double eigenvalue $\lambda_{0}=1.87083 i$ and therefore instability of the IDKN-system by flutter.

\section{Damping in circulatory systems}

In this section we shall study the influence of damping on a general $I K N$-system. As earlier mentioned, the circulatory system is marginally stable if $K+N$ is real symmetrizable with positive eigenvalues and otherwise unstable. An unstable circulatory system can under certain circumstances be marginally stabilized by adding a gyroscopic damping matrix $G$ where $G=-G^{T}$. To show this we use that an $I D G K N$-system wich possesses Hamiltonian symmetry is isospectral with a circulatory $I K_{0} N_{0}$-system through the following relations found in [15]

$$
\begin{aligned}
& K+N=H-H Z H^{-1} Z, \\
& D+G=H Z H^{-1}-Z,
\end{aligned}
$$

where

$$
H=Z^{2}+K_{0}+N_{0}, \quad Z, H, K_{0}, N_{0} \in \mathbb{R}^{n \times n},
$$

and $Z$ and $H$ are non-singular. If $K_{0}+N_{0}$ is symmetrizable and has positive eigenvalues, then all the eigenvalues of the $I D G K N$-system lie on the imaginary axis and the system is marginally stable. We now formulate

\section{Theorem}

An IDGKN-system with Hamiltonian symmetry and a full set of eigenvectors can be generated by Eqs. (11) and (12) where $K_{0}, N_{0}, Z \in \mathbb{R}^{n \times n}$ and $K_{0}+N_{0}$ and $Z$ are chosen such that $Z$ and $H=Z^{2}+K_{0}+N_{0}$ are non-singular. If $K_{0}+N_{0}$ has positive eigenvalues then the IDGKN-system is marginally stable.

Conversely, if an $I D G K N$-system with Hamiltonian symmetry is given then one can find an implicit equation for $K_{0}+N_{0}$ which defines the isospectral $I K_{0} N_{0}$-system expressed by $K+N$ and $D+G$, see [15].

To illustrate the above Theorem, we will show that it is possible to marginally stabilize a circulatory system only by adding a gyroscopic damping $G=-G^{T}$. From (12) we get the condition, $D=0$, for the damping matrix to be skew-symmetric

$$
H^{T}\left(Z+Z^{T}\right) H=H^{T} H Z+Z^{T} H^{T} H .
$$

The above equation is fullfilled if we write $Z=A, A=-A^{T}$ and $H^{T} H=I$. Then Eqs. (11) and (12) leads to

$$
\begin{aligned}
& K+N=H-H A H^{T} A, \quad H^{T} H=I, \quad A=-A^{T}, \\
& G=H A H^{T}-A=-G^{T} .
\end{aligned}
$$

The system is marginally stable if $K_{0}+N_{0}=H+A^{T} A$ is symmetrizable with positive eigenvalues. An example is given below

Example 4.1. If we use the orthogonal matrix

$$
H=\left[\begin{array}{rrrr}
0.656 & 0.231 & 0.552 & 0.459 \\
0.335 & -0.151 & -0.770 & 0.522 \\
-0.512 & -0.507 & 0.301 & 0.625 \\
-0.442 & 0.816 & -0.111 & 0.355
\end{array}\right], \quad H H^{T}=I
$$

and the skew-symmetric matrix

$$
Z=A=\left[\begin{array}{rrrr}
0.00 & -6.00 & -2.20 & -8.90 \\
6.00 & 0.00 & -6.30 & 0.40 \\
2.20 & 6.30 & 0.00 & 2.40 \\
8.90 & -0.40 & -2.40 & 0.00
\end{array}\right]
$$


we find

$$
K_{0}+N_{0}=H-Z^{2}=\left[\begin{array}{rrrr}
121.0 & 10.5 & -58.6 & 8.14 \\
10.6 & 75.7 & 13.4 & 69.0 \\
-59.7 & 13.7 & 50.6 & 17.7 \\
7.24 & 69.3 & 16.9 & 85.5
\end{array}\right]
$$

with the eigenvalues $\{154.0,155.0,12.3,11.1\}$ which shows that the $I K_{0} N_{0}$-system is marginally stable. Further we have according to (15)

$$
K+N=\left[\begin{array}{rrrr}
38.5 & 42.9 & 9.06 & 16.2 \\
76.0 & -16.9 & -27.5 & -25.1 \\
-40.5 & 43.4 & 30.5 & 63.1 \\
-41.6 & 53.2 & 62.3 & 47.4
\end{array}\right]
$$

which has the eigenvalues $\{-23.21,-25.52,74.17 \pm 0.5573 i\}$. Thus the $I K N$-system is unstable. For $G$ we obtain

$$
G=-G^{T}=\left[\begin{array}{rrrr}
0 & 9.105 & -4.862 & 4.297 \\
-9.105 & 0 & 5.470 & -8.695 \\
4.862 & -5.470 & 0 & 1.532 \\
-4.297 & 8.695 & -1.532 & 0
\end{array}\right] \text {. }
$$

The eigenvalues of the system are $\{ \pm 12.44 i, \pm 12.42 i, \pm 3.51 i, \pm 3.33 i\}$. This shows that the $I G K N$-system has Hamiltonian symmetry and is marginally stable.

The above example shows that there exists unstable $I K N$-systems which can be stabilized by adding a gyroscopic damping matrix. But it seems that the procedure does not work for all kind of circulatory systems. Further investigations are necessary to clarify this point.

\section{Conclusions}

Firstly we payed attention to an old stability result of Metelitsyn and Frik for non-conservative systems, since it has been often misunderstood as a necessary condition for stability. Secondly we presented the main goal of this paper, namely the study of some new aspects of circulatory systems. We investigated the influence of indefinite damping on IKNsystems with purely imaginary eigenvalues. Simple examples demonstrated the theory. Systems with for example triple eigenvalues where $K+N$ is not symmetrizable are not taken under consideration. In such a case fractional powers of $\epsilon^{1 / 3}$ in the respective series have to be used. Finally the connection between circulatory systems and more general systems with Hamiltonian symmetry has been addressed with the aim to show how an unstable circulatory system under certain circumstances may be stabilized by gyroscopic damping.

\section{References}

[1] K. Huseyin, Vibrations and Stability of Multiple Parameter Systems (Noordhoff International Publishing, Alphen aan den Rijn, The Netherlands, 1978).

[2] H. Ziegler, Principles of Structural Stability (Birkhäuser Verlag, Basel und Stuttgart, 1977).

[3] P. C. Müller, Stabilität und Matrizen (Springer-Verlag, Berlin, Heidelberg, New York, 1977).

[4] P. Hagedorn, E. Heffel, P. Lancaster, P. C. Müller, and S. Kapuria, Some recent results on MDGKN-systems, ZAMM, Z. Angew. Math. Mech. 95(7), 695-702 (2014).

[5] U. von Wagner, D. Hochlenert, and P. Hagedorn, Minimal models for disk brake squeal, J. Sound Vib. 302, 527-539 (2007).

[6] P. Hagedorn, Neuere Entwicklungen in der technischen Schwingungslehre, ZAMM, Z. Angew. Math. Mech. 68, 22-31 (1988).

[7] R. N. Arnold and L. Maunder, Gyrodynamics and Its Engineering Applications (Academic Press, New York and London, 1961).

[8] I. I. Metelitsyn, The problem of gyroscopic stabilization, Dokl. Akad. Nauk SSSR 86(1), 31-34 (1952).

[9] M. Frik, Zur Stabilität nichtkonservativer linearer Systeme, ZAMM, Z. Angew. Math. Mech. 52, T47-T49 (1972).

[10] W. Kliem and A. P. Seyranian, Metelitsyn's inequality and stability criteria for mechanical systems, J. Appl. Maths. Mechs 68(2), 199-205 (2004).

[11] W. Kliem and P. C. Müller, Gyroscopic stabilization of indefinte damped systems, ZAMM, Z. Angew. Math. Mech. 77(S1), 163-164 (1997).

[12] I. Gohberg, P. Lancaster, and L. Rodman, Matrix Polynomials (Academic Press, San Diego, CA, 1982).

[13] J. Moro, J. V. Burke, and M. L. Overton, On the Lidskii-Vshik-Lyusternik perturbation for eigenvalues of matrices with arbitrary jordan structure, SIAM J. Matrix Anal. Appl. 18(4), 793-817 (1997).

[14] A. P. Seyranian and A. A. Mailybaev, Multiparameter Stability Theory with Mechanical Applications (World Scietific Publishing Co. Pte. Ltd., 2003)

[15] J. Stoustrup, C. Pommer, and W. Kliem, Stability of linear systems in second order form based on structure preserving similarity transformations, ZAMP, Z. Angew. Math. Phys. 66(5), 2909-2919 (2015). 